\title{
TEMPERATURES OF CENTRAL STARS OF PLANETARY NEBULAE
}

\author{
A. PREITE-MARTINEZ \\ Istituto di Astrofisica Spaziale, CP 67, 00044 Frascati, Italy \\ and \\ Observatoire de Strasbourg, 11 rue de l'Universitè, 67000 Strasbourg, France
}

The temperature of Central Stars of Planetary Nebulae (CSPN) is one of the most important parameters for a better understanding of their evolution, their properties and those of the surrounding photoionized nebula. It is an elusive quantity to derive though, because CSPN are generally faint objects and their spectrum is often heavily contaminated by nebular continuum emission.

In the following I will review the different methods used for the determination of the temperature of CSPN, the advances made in this difficult field, and discuss some of the problems arising when comparing temperatures derived with different methods.

\section{Methods}

There are essentially five methods to derive the temperature $\mathrm{T}$ of CSPN, plus nebular models that can be used to get some information on $\mathrm{T}$, plus two scales of calibrated temperatures that can be used as "extrema ratio", e.g. when all the above methods are inapplicable.

\subsection{ZANSTRA}

The Zanstra method (Zanstra, 1931) is quite simple and effective: the number of ionizing photons emitted by the central star can be counted measuring the flux in a single hydrogen recombination line (usually $\mathrm{H}$-beta). A temperature for the central star can be derived if the continuum flux of the star in a given frequency band can also be measured. This procedure yelds the so-called hydrogen Zanstra temperature, $\mathrm{T}_{\mathrm{Z}}(\mathrm{H})$. Similarly, one can use a HeII recombination line to count the number of ionizing photons shortward of the $\mathrm{He}^{+}$threshold, to derive $\mathrm{T}_{\mathrm{Z}}(\mathrm{HeII})$.

Its principal disadvantage is that the continuum of the central star should be observable, and that the nebular spectrum is good enough to give elements for the calculation of the nebular spectrum that has to be subtracted from the observed stellar continuum. Additional problems can arise if the reddening is (very) high or the angular size of the nebula is (very) large.

The basic assumption of the method is that the nebular gas is absorbing all the ionizing photons emitted by the central staí (nebula optically thick in all directions). 
Moreover, the shape of the ionizing continuum has to be selected "a priori": usually that of a blackbody.

The Zanstra method has basically not changed in the last 60 years. Advances in the last five years concerns the number of new applications of the method : Gathier and Pottasch, 1989; Gleizes et al., 1989; Kaler et al., 1989; Pottasch and Acker, 1989; Jacoby and Kaler, 1990; Kaler et al., 1990; Kaler et al., 1991; Tylenda et al., 1991; Kaler and Jacoby, 1991. Apparao and Tarafdar (1989) detected a number of PN with EXOSAT in the energy band $0.05-2.0 \mathrm{KeV}$, and, in an interesting variation of the method, derived $\mathrm{X}$-Ray Zanstra temperatures for $8 \mathrm{CSPN}$. They report a better agreement with $\mathrm{T}_{Z}(\mathrm{HeII})$ than with $\mathrm{T}_{Z}(\mathrm{H})$.

\subsection{ENERGY BALANCE}

The energy-balance (or Stoy) method is based on the assumption that thermal equilibrium holds in the ionized nebular gas, e.g. that energy losses and energy gains exactly balance and define the thermal content of the electron gas in the nebula. The energy-balance (EB) method was also suggested 60 years ago, by Stoy (1933) for a hydrogen nebula. The method was extended by Kaler (1976) to include helium in low-excitation nebulae, and revised and extended by Preite-Martinez and Pottasch (PMP,1983).

The advantage of the EB method is that only the nebular emission spectrum is needed. This is because the energy gain per photoionization (depending on the shape of ionizing continuum of the cantral star) is related to the intensity of all collisionally excited (CE) lines in units of the $\mathrm{H}$-beta intensity. Measuring the central star is not required. The main disadvantage is that strong $\mathrm{CE}$ lines can fall in unobserved (or unobservable) regions of the spectrum. A possible way of estimating the intensity of unseen CE lines was already suggested by PMP. Recently Preite-Martinez et al. $(1989,1991)$ extended this correction scheme to the case of spectra taken in a very limited wavelength region. The correction is rather rough, yet it is the only available. As for the Zanstra method, the spectral distribution of the ionizing star is usually assumed to be that of a black-body.

A nice feature of the EB method is that the derived temperatures are rather insensitive to the optical depth of the nebula, in particular for low-temperature central stars $(\mathrm{T}<$ $50-60.000 \mathrm{~K})$. Nonetheless, the fact that the application of the EB method results in values of the temperature depending on the assumed optical depth status of the nebula, has cast some doubt on the reliability of the method. To remove this drawback, Köppen and Preite-Martinez (1991) have recently thoroughly revised the EB method : with the addition of the observational parameter HeII $4686 / \mathrm{H}_{\beta}$ they derive simultaneously the temperature of the central star and the optical depth of the nebula.

\subsection{IONIC ABUNDANCE RATIOS}

With this method one essentially deduces the shape of the stellar ionizing continuum from the ratio of two consecutive stages of ionization of a given element. Many ratios corresponding to different ionization thresholds are necessary to define the shape of the stellar spectrum down to very short wavelengths. A very good nebular spectrum is the only requirement. After the works of Köppen and Tarafdar (1978) and Natta et al.(1980), no advances have been made.

It is worth noting though that in its simplest form, using the amount of $\mathrm{He}^{++}$relative to $\mathrm{H}^{+}$, the method was suggested by Ambartsumyan (1932) and is often referred to as "cross-over" method (Kaler and Jacoby 1989). 


\subsection{SPECTROSCOPIC ANALYSIS}

The method (Mendez et al. 1988) consists of fitting stellar photospheric $\mathrm{H}$ and He line profiles with theoretical profiles computed from non-LTE models (atmosphere and line formation). The analysis of the observed spectral lines requires sofisticated models, still in refinement. The application of the method is unfortunately limited to relatively right CSPN. Substancial improvements have been made in recent years in spectral resolution, signal-to-noise ratio, sky and nebula subtraction, as well as in non-LTE model atmospheres and line formation codes (Mendez et al. 1988b; Mendez et al. 1990; Gabler et al. 1991; Mendez et al. 1992). All this reflects in the increased reliability of the method.

\subsection{FIT TO CONTINUUM}

The temperature of the observed central star is derived fitting a model for the continuum emission to the observed continuum. The UV wavelength range is used because it is there that the nebular continuum emission is less contaminating the stellar continuum (Bianchi et al. 1986; Bianchi and Grewing 1987). On the other hand, the UV band of hot stars falls in the Rayleigh-Jeans tail of the spectral distribution, reducing the sensitivity of the method at high temperatures. An interesting advance was made recently by Grewing and Neri (1990), who presented a variation of the classical method making use of extinctionindependent UV colour indices.

\subsection{CALIBRATED SCALES}

In addition to the above mentioned methods, one can in principle calibrate an observational quantity against temperatures derived for large samples of CSPN. This approach has been taken by Zijlstra and Pottasch (1989) who tried to calibrate the excitation class of the nebula against the EB temperature of the central star. The fit is fear only for medium excitation nebulae. Kaler and Jacoby (1991) and Mendez (1992) used the observed strength of the OIII 5007 line to find an agreement among the various ways of determining temperatures of central star. Their fits are quite good, in particular at low-temperatures, and can be reliably used for statistical studies of low-excitation nebulae.

\section{Open problems and Discussion}

In the recent past reviewed here, the application of the above methods has led to the determination of the temperature (or of different temperatures referring to the same star) for almost 1000 CSPN. A compilation of all these temperatures has been produced by the author, and its content will be used in the following to discuss the problems that arise when comparing different determinations for the same object.

Because the agreement between temperatures derived with different methods if far from being satisfactory, let's first recall which are the open problems, and use the welth of derived data to try to get some clue on the origin of these problems. Of course a method cannot be better than the assumptions on which it is based, and produce results of better quality than that of the input data. Nonetheless, the answers we are looking for can help us in improving our assumptions, revise methods, and search for more reliable observational data.

The oldest question is: which is the origin of the Zanstra discrepancy? Or, why so many central stars show a $\mathrm{T}_{Z}(\mathrm{H})$ lower than $\mathrm{T}_{Z}(\mathrm{HeII})$ ? In Fig. 1 the Hydrogen versus Helium II Zanstra temperatures are plotted for 133 CSPN with both determinations of the temperature. A well known result shows up clearly: $\mathrm{T}_{Z}(\mathrm{H})$ tends to be lower than 
$\mathrm{T}_{Z}(\mathrm{HeII})$ for $\mathrm{T}_{Z}(\mathrm{H})<100.000 \mathrm{~K}$, almost in agreement above. The ratio $\mathrm{T}_{Z}(\mathrm{HeII}) / \mathrm{T}_{Z}(\mathrm{H})$ (called Zanstra Ratio, $\mathrm{ZR}$ ) can reach values $>2$ at low- $\mathrm{T}$, and it is almost 1 at higher temperatures. Values of the $\mathrm{ZR}<1$ are also present, although not statistically very significant. Another point to note is that in the low- $T$ region there is a continuous distribution between the highest $\mathrm{ZR}$ values and 1 .

We have at least three possible reasons for such a behaviour: (i) we are not counting all the stellar ionizing photons in the H-Lyman continuum, while we are counting all those emitted in the He-Lyman continuum, due to a low nebular optical depth; (ii) our other basic assumption concerning the shape of the far UV continuum emission of the central star is invalid, e.g. the star is not radiating as a black-body; (iii) errors in the determination of the magnitude of the central star. We can immediately rule out reason (iii) as an explanation of the general appearance of Fig.1. The pattern is too well defined to be due to random errors (although they certainly introduce noise); systematic errors (bright stars too brigth, faint stars too faint) could produce such a trend, but how to explain high values of $\mathrm{ZR}$ derived from high quality data?

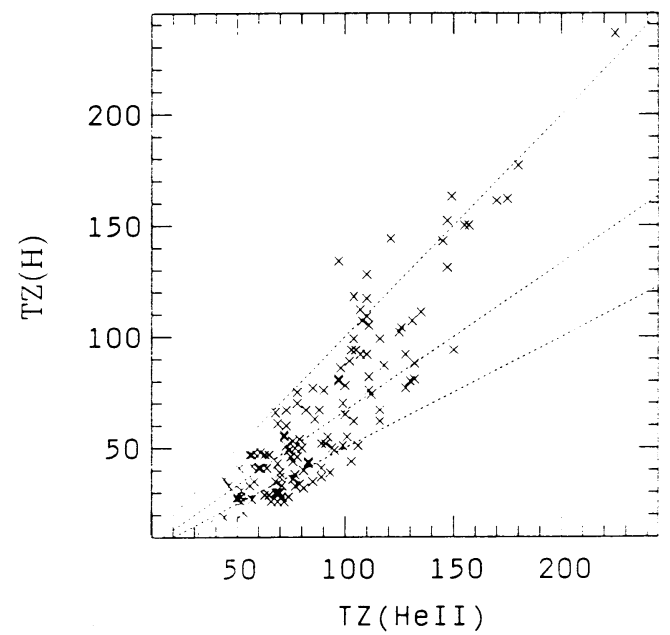

Figure 1. $\mathrm{T}_{Z}(\mathrm{H})$ is plotted versus $\mathrm{T}_{Z}(\mathrm{HeII})$ for 133 CSPN with both determinations of the Zanstra temperature. Dotted lines indicate Zanstra Ratios of 1, 1.5, and 2, clock-wise.

An optical depth effect (i) can easily explain the observed behaviour. In principle, departures from a black-body spectral distribution, alone or coupled with an optical depth effect, could also account for the Zanstra discrepancy. In practice, take the points in Fig.1 around, say, $\mathrm{T}_{Z}(\mathrm{HeII})=70.000 \mathrm{~K}$. We should interpret the range in Zanstra Ratio as due to a continuously changing in the shape of the ionizing continuum of the CSPN. To explain that we need an additional stellar parameter, perhaps gravity. So the adoption of explanation (ii) raises two more questions: (a) is gravity (keeping $\mathrm{T}$ constant) able to influence the shape of the He-Lyman continuum of such an amount as to explain the observed spread in ZR? And (b) do we really observe such a systematic gradient of gravity in Fig.1? Waiting for the answers, we cannot rule out that non-blackbody effects are at work, but certainly the possibility of an optical depth effect gains some strength.

If we now compare Zanstra temperatures with results of direct spectroscopy of the 
central star, we find two features: $\mathrm{T}_{Z}(\mathrm{H})$ is almost always lower than $\mathrm{T}_{M}$ especially for $\mathrm{T}_{M}>40.000 \mathrm{~K}$, and $\mathrm{T}_{Z}(\mathrm{HeII})$ is reasonably well correlated with $\mathrm{T}_{M}$ although frequently higher than $\mathrm{T}_{M}$. The simplest explanation is that $\mathrm{T}_{Z}(\mathrm{H})$ is too low because the nebula is not counting all the ionizing photons: indeed, from the results of the new EB method (Preite-Martinez et al. in preparation) we find that, of the six CSPN for which $\tau$ and $\mathrm{T}_{M}$ are both available, all with $\mathrm{T}_{Z}(\mathrm{H})<\mathrm{T}_{M}$, five are surrounded by very thin nebulae (IC 2448, IC 4593, NGC 1535, NGC 2392, and NGC 7009). A low optical depth cannot be the only mechanism at work in these cases, beacause it cannot explain why the helium Zanstra temperature is often higher than $\mathrm{T}_{M}$. Besides, large errors on stellar magnitudes are improbable, because these CSPN are brigth and well observed.

A point to bear in mind though is that while $\mathrm{T}_{M}$ actually measures the effective temperature of the star assuming that it is radiating as predicted by model atmospheres, the Zanstra temperature could be considered as an effective temperature only if the star was indeed radiating as postulated by the Zanstra method (black-body). If this is not the case, the two methods measure different things, and it should not be surprising that they produce different results.
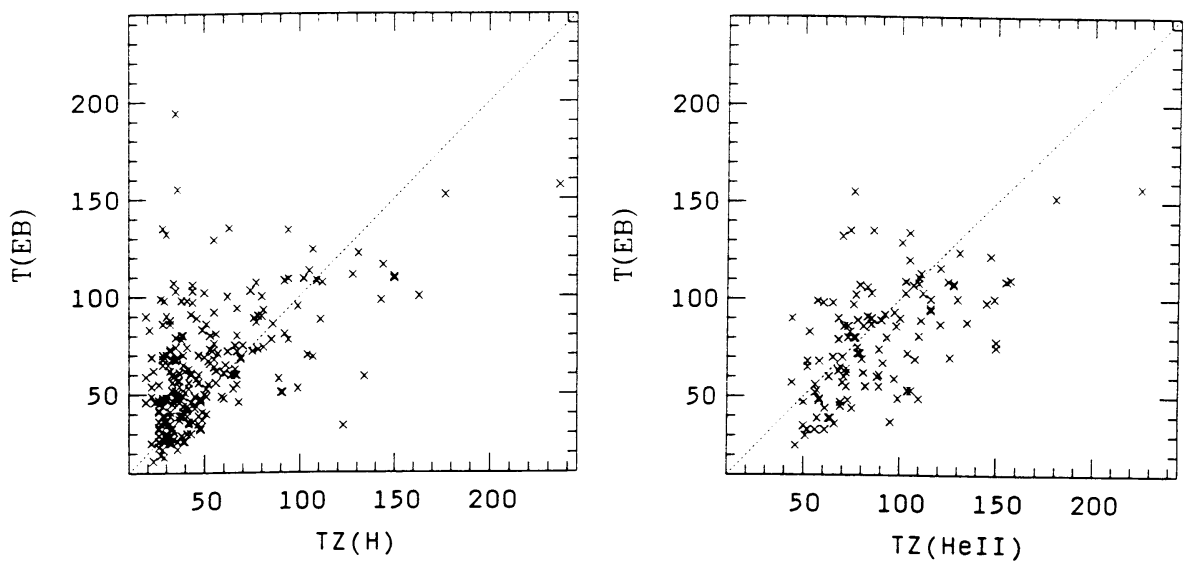

Figure 2. $\mathrm{T}_{E B}$ is plotted versus $\mathrm{T}_{Z}(\mathrm{H})$ (left panel) and $\mathrm{T}_{Z}(\mathrm{HeII})$ (right panel).

An interesting comparison with $\mathrm{T}_{M}$ can be made for another method, also using direct spectroscopic observation of the central star (UV colours). The temperatures $\mathrm{T}_{C}$ so derived are in reasonable agreement with $\mathrm{T}_{M}$ only in about half of the cases. If $\mathrm{T}_{C}$ is compared with $\mathrm{T}_{Z}(\mathrm{H})$, a similar fraction of coincidences if found, the remaining half of the cases being equally spread above or below $\mathrm{T}_{Z}(\mathrm{H})$. A better agreement with $\mathrm{T}_{Z}(\mathrm{HeII})$ is found, but again with a tendency for $\mathrm{T}_{Z}(\mathrm{HeII})$ to be higher than $\mathrm{T}_{C}$. In this case may well be that the major source of disagreement is due to observational errors: indeed the five most discrepant objects are all $\mathrm{PN}$ with small angular diameter, with a continuum emission that can contaminate the stellar continuum (Grewing and Neri, 1990).

We can now move on to discuss the results of the Energy-Balance method. In Fig.2 we plot $\mathrm{T}_{E B}$ versus $\mathrm{T}_{Z}(\mathrm{H})$ and $\mathrm{T}_{Z}(\mathrm{HeII})$. The agreement with Zanstra temperatures is very poor, then we can probably derive important clues. Comparing the two panels, it 
is clear that $\mathrm{EB}$ temperatures are on the average understimated by $15-20 \%$, at least the lowest ones. The most probable reason is that $\mathrm{T}_{E B}$ come mostly from the application of the method to a large sample of nebulae observed in a restricted wavelength region (Preite-Martinez et al. 1989, 1991). The correction scheme to account for unobserved $\mathrm{CE}$ lines probably underestimates the amount of CE cooling in the nebula. Alternatively, or in addition to this, most nebular spectra did not allow the determination of the key parameters necessary to feed the correction scheme (electron temperature and/or density, and ionic abundances of the most important coolants). Again, the quality of the results reflects that of the input data.

Once this effect is taken in due account, we are left with the result that $\mathrm{T}_{E B}$ is always $\geq \mathrm{T}_{Z}(\mathrm{H})$, with very few exceptions. Now, both methods make use of the blackbody assumption, but while the Zanstra method assumes complete optical thickness for the nebula, $\mathrm{T}_{E B}$ is little affected by the optical depth. So what we see in Fig.2 (left panel) is a direct test of the Zanstra's assumption on the optical thickness of the nebulae. The result of the test is that most nebulae are probably optically thin in the H-Lyman continuum.

From the right panel of Fig.2, we get an indirect confirmation because $\mathrm{T}_{E B}$ and $\mathrm{T}_{Z}$ (HeII) roughly correlate, although with a large spread. There could also be an indication that al least part of the scatter is due to observational errors in deriving the magnitude of the central star.

A direct confirmation of our interpretation of Fig.2 comes from Preite-Martinez et al. (in preparation) as the result of the application of the new EB method developed by Köppen and Preite-Martinez (1991). In Fig.3 we plot their new $\mathrm{T}_{E B 2}$ values against $\mathrm{T}_{Z}(\mathrm{H})$, and in the left panel only the points corresponding to optically thin nebulae (optical depth $<3$ ): thin nebulae fall exactly where they should, and the correlation between $\mathrm{T}_{E B}$ and $\mathrm{T}_{Z}(\mathrm{H})$ is much better once the optically thin nebulae are removed.
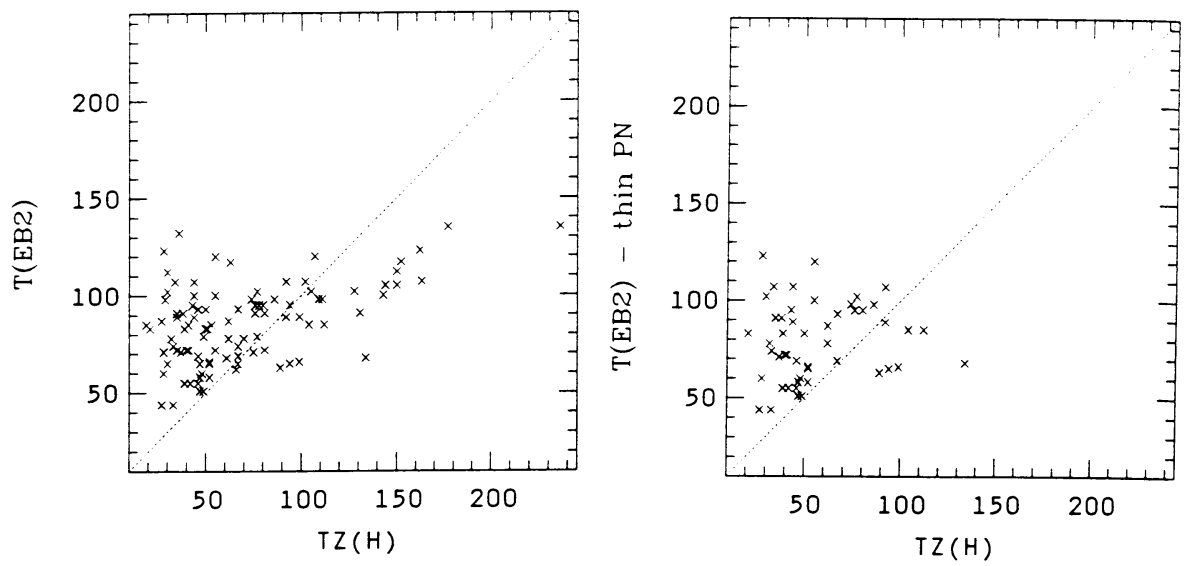

Figure 3. $\mathrm{T}_{E B 2}$ is plotted versus $\mathrm{T}_{Z}(\mathrm{H})$ (left panel). In the right panel only nebulae with $\tau<3$ are plotted.

Another interesting comparison is between $\mathrm{T}_{E B}$ and spectroscopically derived temperatures $\mathrm{T}_{M}$. The agreement is fairly good at low temperatures, beside a few discrepant 
cases of some interest, and it worsens for $\mathrm{T}_{M}$ above $60.000 \mathrm{~K}$. There $\mathrm{T}_{E B}$ is often much higher than $\mathrm{T}_{M}$. What we are actually testing here are two different variations of the assumption on the shape of the ionizing stellar continuum: on one side a blackbody is assumed (EB), the other method uses model atmospheres. Unless we decide "a priori" that one of the two hypothesis is the "true" one, a comparison of this kind can only tell us how to reconcile the results of the two different methods. Indeed, the use of model atmospheres can reduce the values derived with the EB method for hot stars.

Undoubtably, one of the most promising advances recently made in this field is the possibility of deriving the optical depth of the nebula with the new version of the EB method proposed by Köppen and Preite-Martinez (1991). The application of the method to about 360 objects by Preite-Martinez et al., still unpublished, will open the possibility of a direct test on one of the most criticized assumptions: that planetary nebulae are mostly optically thick in the H-Lyman continuum. We can anticipate some of the new results on the determination of the optical depth $\tau$ : half of the nebulae examined are optically thin $(\tau<3)$. In Table 1 we extracted from our compilation of recent results those nebulae with at least 4 different determinations of the temperature of the central star (both Zanstra temperatures plus two other, or $\mathrm{T}_{Z}(\mathrm{H})$ only, plus three other temperatures). Although the sample is small, the trend is clear: to thin nebula always correspond a $\mathrm{T}_{Z}(\mathrm{HeII})>$ $\mathrm{T}_{Z}(\mathrm{H})$. Conversely, the Zanstra Ratio $\mathrm{ZR}$ correlates very well with $\tau$ (beside M 3-6, whose $\mathrm{T}_{Z}(\mathrm{H})$ is unreliable, and NGC 3242).

Table 1 - CSPN with at least 4 different determinations of the temperature (both Zanstra plus 2 others, or $\mathrm{T}_{Z}(\mathrm{H}), \mathrm{T}_{M}, \mathrm{~T}_{C}$ and $\left.\mathrm{T}_{E B}\right)$.

\begin{tabular}{|c|c|c|c|c|c|c|c|c|}
\hline PK & Name & $\mathrm{T}_{Z}(\mathrm{H})$ & $\mathrm{T}_{Z}(\mathrm{HeII})$ & $\mathrm{T}_{M}$ & $\mathrm{~T}_{C}$ & $\mathrm{~T}_{E B}$ & $\mathrm{~T}_{E B 2}$ & $\log (\tau)$ \\
\hline $25+401$ & IC 4593 & $28 a$ & 50 & 40 & 35 & $47 a$ & 60 & 0.29 \\
\hline $34+111$ & NGC 6572 & 66 & - & 60 & 34 & 60 & - & - \\
\hline $37-341$ & NGC 7009 & 60 & 84 & 82 & 51 & 73 & 78 & 0.70 \\
\hline $54-121$ & NGC 6891 & $35 a$ & - & 50 & 31 & $52 a$ & - & - \\
\hline $43+371$ & NGC 6210 & $61 \mathrm{a}$ & $69 a$ & - & 37 & $65 a$ & 68 & 1.96 \\
\hline $83+121$ & NGC 6826 & 33 & - & 50 & 42 & $33 a$ & - & - \\
\hline $123+341$ & IC 3568 & 31 & 52 & 50 & 59 & $65 a$ & - & - \\
\hline $197+171$ & NGC 2392 & 27 & 67 & 47 & 33 & 118 & 105 & 0.79 \\
\hline $206-401$ & NGC 1535 & 35 & 68 & 70 & 63 & 79 & 89 & 0.59 \\
\hline $215-241$ & IC 418 & 38 & - & 36 & 39 & $29 a$ & - & - \\
\hline $254+51$ & M 3-6 & 48: & 58 & - & 43 & 48 & 60 & 0.24 \\
\hline $261+321$ & NGC 3242 & 54 & 91 & 75 & 50 & 109 & 100 & 1.36 \\
\hline $285-141$ & IC 2448 & 43 & 83 & 65 & 72 & 91 & 95 & 0.06 \\
\hline $316+81$ & He 2-108 & $26 a$ & 52 & 33 & - & 33a & - & - \\
\hline $327+101$ & NGC 5882 & $55 \mathrm{a}$ & $72 a$ & - & 32 & $64 a$ & 72 & 0.77 \\
\hline $345-81$ & Tc 1 & $30 a$ & - & 33 & 33 & 34 & - & - \\
\hline
\end{tabular}

$\mathrm{a}=$ average value; $\mathrm{T}$ in $10^{3} \mathrm{~K}$.

We have seen in the discussion above that comparing results of different methods is sometimes a good way of testing assumptions and the methods themselves. Model atmospheres are certainly to be improved, and the correction scheme that is used to estimate the total CE cooling also needs improvement or revision. Observational errors still play a non 
negligible role in all methods. Piece by piece the picture is getting clearer: a good fraction of planetary nebulae must be thin to explain most of the discrepancies between the results of different methods. According to Preite-Martinez and collaborators a determination of the optical depth is now available for a large sample of medium/high-excitation nebulae, and half of them are indeed thin. Departures from blackbody distribution cannot be ruled out, but have little statistical weight.

\section{References}

Ambartsumyan, V. A. 1932, Pulkovo Obs. Circ. Vol.8, No.4

Apparao, K. M. V., Tarafdar, S. P. 1989, Astrophys. J. 344, 826

Bianchi, L., Cerrato, S., Grewing, M. 1986, Astron. Astrophys. 169, 227

Bianchi, L., Grewing, M. 1987, Astron. Astrophys. 181, 85

Gabler, R., Kudritzki, R. P., Mendez, R. H. 1991, Astron. Astrophys. 245, 587

Gathier, R., Pottasch, S. R. 1989, Astron. Astrophys. 209, 369

Gleizes, F., Acker, A., Stenholm, B. 1989, Astron. Astrophys. 222, 237

Grewing, M., Neri, R. 1990, Astron. Astrophys. 236, 223

Jacoby, G. H., Kaler, J. B. 1989, Astron. J. 98, 1662

Kaler, J. B. 1976 Astrophys. J. 210, 843

Kaler, J. B., Jacoby, G. H. 1989, Astrophys. J. 345, 871

Kaler, J. B., Jacoby, G. H. 1991, Astrophys. J. 372, 215

Kaler, J. B., Shaw, R. A., Feibelman, W. A., Lutz, J. H. 1989, Astrophys. J. Suppl. Ser. 70, 213

Kaler, J. B., Shaw, R. A., Kwitter, K. B. 1990, Astrophys. J. 359, 392

Kaler, J. B., Shaw, R. A., Feibelman, W. A., Imhoff, C. L. 1991, Publ. Astron. Soc. Pac. 103, 67

Köppen, J., Tarafdar, S. P. 1978, Astron. Astrophys. 69, 363

Köppen, J., Preite-Martinez, A. 1991, Astron. Astrophys. 248, 191

Mendez, R. H., Kudritzki, R. P., Herrero, A., Husfeld, D., Groth, H. G. 1988, Astron. Astrophys. 190, 113

Mendez, R. H., Groth, H. G., Husfeld, D., Kudritzki, R. P., Herrero, A. 1988b, Astron. Astrophys. 197, L25

Mendez, R. H., Herrero, A., Manchado, A. 1990, Astron. Astrophys. 229, 152

Mendez, R. H. 1992, Astron. Astrophys., in press

Mendez, R. H., Kudritzki, R. P., Herrero, A. 1992, Astron. Astrophys., in press

Natta, A., Pottasch, S. R., Preite-Martinez, A. 1980, Astron. Astrophys. 84, 284

Pottasch, S. R., Acker, A. 1989, Astron. Astrophys. 221, 123

Preite-Martinez, A., Pottasch, S. R. 1983, Astron. Astrophys. 126, 31

Preite-Martinez, A., Köppen, J., Acker, A., Stenholm, B. 1989, Astron. Astrophys. Suppl. Ser. 81, 309

Preite-Martinez, A., Köppen, J., Acker, A., Stenholm, B. 1991, Astron. Astrophys. Suppl. Ser. 88, 121

Stoy, R. H. 1933, Montly Notices Roy. Astron. Soc. 93, 588

Tylenda, R., Stasinska, G., Acker, A., Stenholm, B. 1991, Astron. Astrophys. 246, 221

Zanstra, H. 1931, Publ. Dom. Astroph. Obs. 4, 209

Zijlstra, A. A. and Pottasch, S. R. 1989 Astron. Astrophys. 216, 245 